\title{
RELATiOnShip Between OPTIMAL LACTATE Removal Power Output and Olympic Triathlon Performance
}

\author{
Carlo Baldari, ${ }^{1}$ Luigi Di Luigi, ${ }^{1}$ Sergio G. Da Silva, ${ }^{2}$ Maria C. Gallotta, ${ }^{1}$ \\ Gian P. Emerenziani, ${ }^{1}$ Caterina Pesce, ${ }^{3}$ and Laura Guidetti ${ }^{1}$
}

\author{
${ }^{1}$ Department of Health Sciences and ${ }^{3}$ Department of Educational Sciences for Motor Activities and Sports, \\ University of Rome "IUSM," Rome, Italy; ${ }^{2}$ Department of Physical Education, Federal University of Paraná \\ (UFPR), Curitiba, Brazil.
}

\begin{abstract}
Baldari, C., L. Di Luigi, S.G. Da Silva, M.C. Gallotta, G.P. Emerenziani, C. Pesce, and L. Guidetti. Relationship between optimal lactate removal power output and Olympic triathlon performance. J. Strength Cond. Res. 21(4):1160-1165. 2007.-To investigate the relationships between race performance and parameters at the optimal power output for lactate removal, 10 male triathletes were examined. Exercise intensities for lactate removal were defined by calculating $50 \%$ of difference $(\Delta \mathrm{T})$ between running velocity $\left(\mathrm{V}_{\mathrm{r}}\right)$ at individual anaerobic threshold (IAT) and at individual ventilatory threshold (IVT), then choosing $3 \mathrm{~V}_{r}$ : at IVT plus $50 \% \Delta \mathrm{T}\left(\mathrm{IVT}_{+50 \% \Delta \mathrm{T}}\right)$, at IVT, and at IVT minus $50 \% \Delta \mathrm{T}\left(\mathrm{IVT}_{-50 \% \Delta \mathrm{T}}\right)$. After a 6 -minute treadmill run at $75 \%$ of difference between IAT and $\dot{\mathrm{V}}_{2}$ max, all triathletes performed a 30 -minute active recovery run at $\mathrm{IVT}_{+50 \% \Delta \mathrm{T}}$, IVT, and IVT ${ }_{-50 \% \Delta \mathrm{T}}$. Capillary blood lactate was determined at $1,3,6$, $9,12,15,20,25$, and 30 minutes of recovery. The $\mathrm{IVT}_{-50 \% \Delta \mathrm{T}}$ recovery was the most efficient $\mathrm{V}_{\mathrm{r}}$ for lactate removal. Running velocities at IVT and $\mathrm{IVT}_{-50 \% \Delta \mathrm{T}}$ were highly $(p<0.01)$ related to cycle, run, and overall race time. $\dot{\mathrm{VO}}_{2}$ values at IAT, $\mathrm{IVT}_{+50 \% \Delta \mathrm{T}}$, and IVT were less $(p<0.05)$ related to split and overall race time. The variable most related to overall race time, as determined by stepwise multiple linear regression analysis, was the $\mathrm{V}_{\mathrm{r}}$ at $\operatorname{IVT}_{-50 \% \Delta \mathrm{T}}(r=0.87, p=0.001)$. The $R^{2}$ value of 0.76 indicated that $\mathrm{V}_{\mathrm{r}}$ at $\mathrm{IVT}_{-50 \% \Delta \mathrm{T}}$ could account for $76 \%$ of the variance in triathlon race time. This study shows that the race performances of triathletes are highly related to the $\mathrm{V}_{\mathrm{r}}$ at which the most efficient lactate removal (IVT ${ }_{-50 \% \Delta \mathrm{T}}$ ) occurs. These findings suggest that the assessment of $\mathrm{V}_{\mathrm{r}}$ at IVT and IAT (from which $\mathrm{V}_{\mathrm{r}}$ at $\mathrm{IVT}_{-50 \% \Delta \mathrm{T}}$ are calculated) may be a useful method for monitoring training-induced adaptations and performance improvements in athletes who participate in Olympic triathlons.
\end{abstract}

KEY WoRDs. lactate metabolism, treadmill ergometer, running, male triathletes

\section{INTRODUCTION}

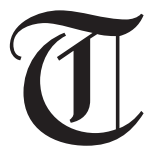

he triathlon is a multisport event consisting of sequential swimming, cycling, and running over "long" or "short" distances. The distances of each competition segment may vary substantially, so that the total competition time ranges from 30 minutes to several hours $(25,26)$. In particular, the standard distance (also known as "short distance" and "Olympic triathlon") consists of 1,500 m of swimming, $40 \mathrm{~km}$ of cycling, and $10 \mathrm{~km}$ of running.

A previous study (12) demonstrated an unexpected rise in blood lactate $\left(8.1 \mathrm{mmol} \cdot \mathrm{L}^{-1}\right)$ after the first segment (swimming) followed by declining levels after the other 2 segments (5.1 and $4.4 \mathrm{mmol} \cdot \mathrm{L}^{-1}$ cycling and running, respectively). This lactate trend during a prolonged effort, such as the Olympic triathlon, has been related with an early appearance of ventilatory threshold and an impair- ment of performance after swimming and cycling (8). The correlation between the ventilatory threshold and the endurance performance is well known; in fact, for triathlon performance the $\dot{\mathrm{VO}}_{2}$ at ventilatory threshold was reported to be a better predictor than $\mathrm{VO}_{2} \max$ (30) for both running time and total time $(8,31)$.

During a triathlon event, blood lactate was observed to decrease progressively after the first segment. The lactate decrement was observed to occur regardless of the order of segments (12). It is important to remember that lactate removal is accomplished mainly through oxidation within mitochondria of active well-oxygenated red muscles, as suggested by the lactate shuttle hypothesis of Brooks (6). Whether lactate can be oxidized by working muscles depends on the intensity of the exercise, because different intensities can lead to different muscle behavior from uptake to output of lactate $(10,13)$. Thus, because lactate can be regarded as an energy substrate for working muscles during competition, particularly competition of a long duration, a high exercise intensity during which the most efficient lactate removal occurs could be considered a positive factor for successful performance. This is because whenever exercise intensity is not efficient for lactate removal, the resulting lactate accumulation is associated with an attenuate adenosine triphosphate production that inhibits the glycolytic rate-limiting enzymes and decreases the lipolysis rate (14); lactate accumulation is also associated with a rise in muscle and blood $\mathrm{H}^{+}$concentration, which has been related directly (19) or indirectly (27) to local fatigue. Moreover, the lactate anion can cause fatigue in skeletal muscle independent of associated reductions in $\mathrm{pH}(15)$.

We recently studied the optimal lactate removal power output in soccer players (2), quantifying the exercise intensity not in percent of $\mathrm{V}_{2}$ max but in relation to validated metabolic reference points (23), such as the individual anaerobic threshold (IAT) and the individual ventilatory threshold (IVT). In this study, we wanted to determine whether the power output at which the most efficient lactate removal occurs is related to short-distance triathlon performance.

\section{Methods}

\section{Experimental Approach to the Problem}

This study was designed to determine the relationship between power output for optimal lactate removal and performance during an Olympic triathlon. For this purpose, we verified, in laboratory conditions, the effect of some exercise intensities below the IAT calculated in re- 
lation to the IAT and the IVT. As previously reported for studying lactate removal (2), 3 recovery intensities were considered: running velocity $\left(\mathrm{V}_{\mathrm{r}}\right)$ at (a) IVT, (b) middle intensity between IVT and IAT, i.e., at $50 \%$ of IAT minus IVT $(\Delta \mathrm{T})$ above IVT $\left(\mathrm{IVT}_{+50 \% \Delta \mathrm{T}}\right.$ ), and (c) $50 \% \Delta \mathrm{T}$ below the IVT $\left(\mathrm{IVT}_{-50 \% \Delta \mathrm{T}}\right)$. We then investigated the relationship between race time recorded during a triathlon competition and such laboratory variables as $\mathrm{VO}_{2}$ and $\mathrm{V}_{\mathrm{r}}$ measured at $\mathrm{IVT}_{+50 \% \Delta \mathrm{T}}$ IVT and IVT ${ }_{-50 \% \Delta \mathrm{T}}$ power outputs.

\section{Subjects}

Ten triathlon male athletes participated in the study. After approval was received from the institute's Ethics Committee, subjects gave their written informed consent. Their mean $( \pm S D)$ physical characteristics were as follows: height, $1.81 \pm 0.03 \mathrm{~m}$; body mass, $73 \pm 3 \mathrm{~kg}$; age, $24 \pm 3$ years; $\mathrm{V}_{2} \max , 70.7 \pm 4.6 \mathrm{~mL} \cdot \mathrm{kg}^{-1} \cdot \mathrm{min}^{-1} ; \mathrm{VO}_{2 \mathrm{IAT}}$, $53.6 \pm 3.9 \mathrm{~mL} \cdot \mathrm{kg}^{-1} \cdot \mathrm{min}^{-1} ; \quad \mathrm{VO}_{2 \mathrm{IVT}}, \quad 43.2 \pm 4.7$ $\mathrm{mL} \cdot \mathrm{kg}^{-1} \cdot \mathrm{min}^{-1}$. All subjects had been training regularly for approximately 12-15 hours a week for at least the previous 3 years.

All subjects were in good health and were not taking any medications, amino acids, or other drugs, including anabolic doping agents that would affect the results of this study. None of the subjects was a smoker. All subjects were counseled by a nutritionist and had a diet regimen sufficient for their individual needs (44-50 $\mathrm{kcal} \cdot \mathrm{kg}^{-1} \cdot \mathrm{d}^{-1}: 55-60 \%$ carbohydrates, $15-20 \%$ proteins, and $\sim 25 \%$ lipids). This diet regimen started 2 weeks before the experimental phases and was maintained throughout the study. The subjects were counseled weekly and their food records reviewed to maintain the correct diet and fluid consumption throughout the study. These guidelines recommended that all subjects maintain their food intake and remain well hydrated, especially before each test. They also maintained their habitual sleep patterns.

\section{Detecting Optimal Lactate Removal Power Output}

Subjects performed a treadmill presession test to evaluate the $\mathrm{VO}_{2} \max$, IAT, and IVT. Subsequently, subjects performed in random order 3 experimental recovery trials on treadmill on 3 separate occasions. A minimum of 48 hours separated each exercise test; the tests took place over 3 consecutive weeks and ended 2 weeks before the national triathlon competition. They began at about the same time of day for a given subject (between 8:30 and 10:30 AM), and the environmental conditions were always identical (temperature $21-22^{\circ} \mathrm{C}$; humidity $50-60 \%$ ). The subjects were requested to have breakfast (about the same $\mathrm{kcal} \cdot \mathrm{kg}^{-1}$ and nutrient composition) 2 hours before the experimental test. No intensive training was allowed on the day before each test.

Presession Test. To determine the $\mathrm{VO}_{2} \max$, IVT, and IAT, each subject performed a continuous, graded treadmill test. The treadmill maximal test consisted of a 3-minute walking warm-up at $6 \mathrm{~km} \cdot \mathrm{h}^{-1}$ with $0 \%$ slope, followed by a velocity increment of $2 \mathrm{~km} \cdot \mathrm{h}^{-1}$ every 3 minutes up to the power output subsequent to IAT (1). Then a $3 \%$ increment in slope every minute was given. When the $\dot{\mathrm{V}}_{2}$ max was obtained, an active recovery of 1 minute walking at $6 \mathrm{~km} \cdot \mathrm{h}^{-1}$ with $0 \%$ slope followed. $\dot{\mathrm{VO}}_{2} \max$ was identified at the occurrence of a plateau of $\mathrm{VO}_{2}$, despite a further increase in power output or an increase in $\mathrm{V}_{2}<1$ $\mathrm{mL} \cdot \mathrm{kg}^{-1} \cdot \mathrm{min}^{-1}$ in comparison with that produced by the previous power output (11). Secondary criteria were also applied to verify the maximal effort, such as an attain- ment of age-predicted maximum heart rate and/or a respiratory exchange ratio $>1.15(11) . \dot{V}_{2}, \dot{V}_{E}$, and heart rate were acquired breath by breath and then averaged every 30 seconds during the test by a telemetric gas analysis system (K4 COSMED, Rome, Italy) that has been shown to be valid and reliable (24).

To determine the oxygen consumption at ventilatory (IVT) and lactate (IAT) thresholds, the average of the last minute of each corresponding level was used. Determination of blood lactate was carried out using capillary blood from a fingertip. Blood lactate concentration was immediately analyzed during the treadmill test using an Accusport lactate analyzer (Boehringer Mannheim, Mannheim, Germany) that has been shown to be valid and reliable (4). Blood lactate evaluations were performed at the third minute of a given power output for each work load until the power output subsequent to that corresponded to the IAT.

The IVT was determined as the level of $\mathrm{VO}_{2}$ at which the minimum value of the ventilatory equivalent of oxygen $\left(\dot{\mathrm{V}}_{\mathrm{E}} / \dot{\mathrm{V}}_{2}\right)$ calculated from an individual $\dot{\mathrm{V}}_{2}$ vs. $\dot{\mathrm{V}}_{\mathrm{E}} / \dot{\mathrm{VO}}_{2}$ plot was observed $(2,16)$. To determine the IAT, we used a simplified method previously validated (1). The IAT was determined plotting an individual curve of $\mathrm{VO}_{2}$ vs. lactate. In this curve, each lactate value was assigned to the power output immediately prior to that of its measurement, and consequently the individual $\mathrm{V}_{2}$ vs. lactate curve was plotted (1). The IAT was defined as the power output corresponding to the second lactate increase of at least 0.5 $\mathrm{mmol} \cdot \mathrm{L}^{-1}$ from the previous value, where the second increase was greater than (or equal to) the first one (1).

Experimental Recovery Trials. To determine the power output for optimal lactate removal, 3 recovery exercise intensities below the IAT (2) were investigated: (a) IVT, (b) $\mathrm{IVT}_{+50 \% \Delta \mathrm{T}}$, and (c) $\mathrm{IVT}_{-50 \% \Delta \mathrm{T}}$. Trial sequences were randomized to eliminate bias. Each recovery trial consisted of a warm-up of 3 minutes of walking at $6 \mathrm{~km} \cdot \mathrm{h}^{-1}$ then a 6 -minute run at $75 \%$ of the difference between the IAT and the individual $\mathrm{V}_{2}$ max. This constant load exercise corresponded to $90 \% \mathrm{VO}_{2}$ max previously utilized to study lactate removal $(2,22)$. Immediately at the end of this intense exercise, the desired recovery power output was set and maintained for 30 minutes. The IVT power output was the $V_{\mathrm{r}}$ at which the IVT $\left(\mathrm{V}_{\mathrm{r} \text { IVT }}\right)$ was found. The $\mathrm{IVT}_{+50 \% \Delta \mathrm{T}}$ power output was defined as the $\mathrm{V}_{\mathrm{r}}$ halfway between the $V_{r}$ at IVT and $V_{r}$ at IAT. The IVT I $_{-50 \% \Delta T}$ power output was defined as the $V_{r}$ at IVT minus the half-difference between the $\mathrm{V}_{\mathrm{r}}$ at IAT and $\mathrm{V}_{\mathrm{r}}$ at IVT.

During each recovery trial, blood lactate measurements were performed at rest and at 1, 3, 6, 9, 12, 15, 20, 25 , and 30 minutes of exercise recovery without running interruptions.

\section{Triathlon Race Performance}

Within 2 weeks after the last laboratory test, all subjects competed in the national triathlon championship. The weather conditions during the race were $20.6^{\circ} \mathrm{C}, 52 \% \mathrm{hu}$ midity, and a wind speed of $2.9 \mathrm{~m} \cdot \mathrm{s}^{-1}$. The short-distance race was held at sea level on a flat out and back course and consisted of a 1,500-m pool swim, a 40-km cycle, and $10-\mathrm{km}$ run. Drafting was allowed. The time to complete the race was recorded by the race organizers to the nearest second.

\section{Statistical Analyses}

For each subject and each recovery condition, a blood lactate recovery curve as a percentage of accumulated lac- 
TABLE 1. Swim, cycle, run, and overall triathlon performance times for 10 male triathletes.

\begin{tabular}{|c|c|c|c|c|}
\hline & 1,500-m swim & 40-km cycle & 10-km run & Overall time \\
\hline Mean $\pm S D(\min : \mathrm{s})$ & $23: 13 \pm 1: 47$ & $62: 58 \pm 2: 23$ & $37: 50 \pm 2: 28$ & $124: 01 \pm 6: 12$ \\
\hline Range (min:s) & $20: 18-26: 35$ & $59: 29-66: 14$ & $34: 50-41: 8$ & $116: 17-132: 57$ \\
\hline Correlation with overall time & $0.74^{*}$ & $0.90 * *$ & $0.94^{* *}$ & \\
\hline
\end{tabular}

$* r \geq 0.63, p \leq 0.05 ; * * r \geq 0.75, p \leq 0.01$.

TABLE 2. Running velocity $\left(\mathrm{V}_{\mathrm{r}}\right)$ and oxygen consumption $\left(\mathrm{V}_{2}\right)$ at selected submaximal power outputs.

\begin{tabular}{lrrrr}
\hline & \multicolumn{1}{c}{ IAT $^{*}$} & \multicolumn{1}{c}{ IVT $_{+50 \% \Delta \mathrm{T}}$} & \multicolumn{1}{c}{ IVT $_{-50 \% \Delta \mathrm{T}}$} \\
\hline $\mathrm{V}_{\mathrm{r}}\left(\mathrm{m} \cdot \mathrm{s}^{-1}\right)$ & $4.4 \pm 0.4$ & $3.8 \pm 0.4$ & $3.2 \pm 0.4$ & $2.6 \pm 0.5$ \\
$\mathrm{VO}_{2}\left(\mathrm{~mL} \cdot \mathrm{kg}^{-1} \cdot \mathrm{min}^{-1}\right)$ & $53.6 \pm 4.0$ & $47.8 \pm 4.4$ & $43.2 \pm 4.7$ & $38.1 \pm 5.0$ \\
\hline
\end{tabular}

* Values are mean $\pm S D$. IAT $=$ individual anaerobic threshold; IVT $=$ individual ventilatory threshold.

tate $\left(\mathrm{mmol} \cdot \mathrm{L}^{-1}\right)$ vs. time (minutes) was plotted. Results are reported as mean $\pm S D$. Analysis of variance for repeated measures was used to detect significant effects of the recovery power output $\left(\mathrm{IVT}_{+50 \% \Delta \mathrm{T}}, \mathrm{IVT}, \mathrm{IVT}_{-50 \% \Delta \mathrm{T}}\right.$ ). Posthoc analysis of significant differences was performed using the Student-Newman-Keuls test. A significance level of $p \leq 0.05$ was selected.

Pearson's Product moment correlations describe the relationship between the individual physiological variables measured and race performance in each phase of the triathlon, as well as overall race performance time for all triathletes. A stepwise multiple linear regression analysis was used to determine the physiological variables most related to overall race time. Correlation coefficients of $|r|=0.63$ and $|r|=0.75$ corresponded to $\alpha=0.05$ and $\alpha=0.01$ with a statistical power of 0.76 and 0.95 , respectively.

\section{RESULtS}

\section{Triathlon Race Performance}

Table 1 shows the mean overall race time along with the splits for each segment of the race. The corresponding correlation coefficients for each segment of the race vs. the overall race time are also displayed. The race time for both cycle and run sections was highly related to total race time ( $r=0.94$ and $r=0.90, p<0.01$, respectively). Also, the swimming time was significantly correlated to the overall competition time $(r=0.74, p<0.05)$.

\section{Optimal Lactate Removal Power Output}

Time courses of blood lactate concentration during recovery at $\mathrm{IVT}_{+50 \% \Delta \mathrm{T}}$ IVT, and $\mathrm{IVT}_{-50 \% \Delta \mathrm{T}}$ exercise intensities were significantly different $(p<0.001)$ (Figure 1$)$. In particular, the lactate removal showed a similar curve at IVT $_{+50 \% \Delta \mathrm{T}}$ and IVT until the $20^{\text {th }}$ minute. At IVT ${ }_{-50 \% \Delta \mathrm{T}}$, the lactate removal curve presented significantly lower values vs. the other 2 exercise intensities from the ninth to $30^{\text {th }}$ minute.

\section{Relationship Between Race Performance and Laboratory Parameters}

Selected submaximal physiological variables are reported in Table 2. These variables include $\mathrm{V}_{\mathrm{r}}$ and $\mathrm{V}_{2}$ values at IAT, $\mathrm{IVT}_{+50 \% \Delta \mathrm{T}}, \mathrm{IVT}$, and $\mathrm{IVT}_{-50 \% \Delta \mathrm{T}}$. The interrelationships among these variables and race performance variables are displayed in Table 3 as correlation coefficients. $\dot{\mathrm{V}}_{2}$ values at IAT were significantly $(p<0.05)$ related to cycle, run, and overall race time. $\mathrm{VO}_{2}$ values at $\mathrm{IVT}_{+50 \% \Delta \mathrm{T}}$ and IVT were significantly $(p<0.05)$ correlated to overall race time. The $\mathrm{V}_{\mathrm{r}}$ at IAT was only significantly $(p<0.05)$

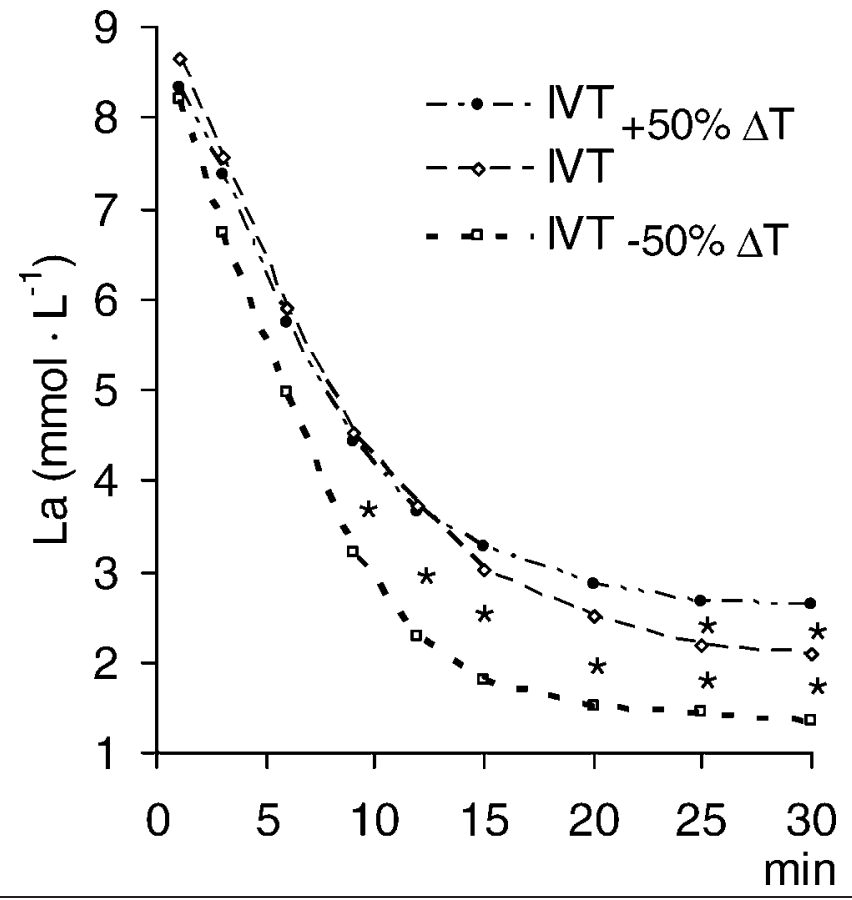

FigURE 1. Blood lactate curves comparison among 3 recovery conditions $\left(\mathrm{IVT}_{+50 \% \Delta \mathrm{T}}, \mathrm{IVT}, \mathrm{IVT}_{-50 \% \Delta \mathrm{T}}\right)$ in triathlon athletes. IVT $=$ individual ventilatory threshold. * indicates a significant difference between recovery blood lactate curves at a given time point.

related to running race time. The $\mathrm{V}_{\mathrm{r}}$ at $\mathrm{IVT}_{+50 \% \Delta \mathrm{T}}$ was correlated to cycle, run, and overall race time $(p<0.05, p<$ 0.01 , and $p<0.05$, respectively). The $\mathrm{V}_{\mathrm{r}}$ at IVT and IVT $_{-50 \% \Delta \mathrm{T}}$ were highly $(p<0.01)$ related to cycle, run, and overall race time. None of the selected submaximal physiological variables was correlated with the swim race time. The submaximal variable most related to overall race time, as determined by stepwise multiple linear regression analysis, was the running velocity at $\mathrm{IVT}_{-50 \% \Delta \mathrm{T}}$ $(r=0.87, p=0.001)$. The $R^{2}$ value of 0.76 indicated that this laboratory measure could account for $76 \%$ of the variance in triathlon race time (Figure 2).

\section{Discussion}

The physical characteristics (age, weight, height) of triathletes in the present study were similar to those previously reported for male triathletes $(8,25,28)$. $\mathrm{VO}_{2} \max$ values during treadmill running were similar to those recently reported for elite triathletes $(17,25,28)$. It is already known that the wide range in $\mathrm{VO}_{2} \max$ (from 52 to 
TABLE 3. Correlations of split and overall race times with running velocity $\left(\mathrm{V}_{\mathrm{r}}\right)$ and oxygen consumption $\left(\mathrm{VO}_{2}\right)$ at selected submaximal power outputs.*

\begin{tabular}{|c|c|c|c|c|c|}
\hline & & 1,500-m swim & 40-km cycle & $10-\mathrm{km}$ run & Overall time \\
\hline \multirow[t]{4}{*}{$\overline{V_{r}\left(m \cdot s^{-1}\right)}$} & IAT & -0.26 & -0.48 & $-0.63 \dagger$ & -0.59 \\
\hline & $\mathrm{IVT}_{+50^{\circ} \mathrm{TT}}$ & -0.39 & $-0.69 \dagger$ & $-0.77 \ddagger$ & $-0.72 \dagger$ \\
\hline & $\mathrm{IVT}^{+50 \% \Delta \mathrm{T}}$ & -0.47 & $-0.81 \ddagger$ & $-0.91 \ddagger$ & -0.85 \\
\hline & IVT $_{-50 \% \Delta \mathrm{T}}$ & -0.49 & $-0.82 \ddagger$ & $-0.93 \ddagger$ & $-0.87 \ddagger$ \\
\hline \multirow[t]{4}{*}{$\dot{\mathrm{V}} \mathrm{O}_{2}\left(\mathrm{~mL} \cdot \mathrm{kg}^{-1} \cdot \mathrm{min}^{-1}\right)$} & $\mathrm{IAT}^{-50 \% \Delta \mathrm{T}}$ & -0.47 & $-0.67 \dagger$ & $-0.63 \dagger$ & $-0.69 \dagger$ \\
\hline & $\mathrm{IVT}_{+50 \% \Delta \mathrm{T}}$ & -0.55 & -0.61 & -0.61 & $-0.68 \dagger$ \\
\hline & IVT & -0.48 & -0.58 & -0.60 & $-0.64 \dagger$ \\
\hline & $\mathrm{IVT}_{-50 \% \Delta \mathrm{T}}$ & -0.41 & -0.56 & -0.57 & -0.60 \\
\hline
\end{tabular}

$*$ IAT $=$ individual anaerobic threshold; IVT $=$ individual ventilatory threshold. Significant correlations: $\dagger|\mathrm{r}| \geq 0.63, p \leq 0.05 ; \ddagger|\mathrm{r}|$ $\geq 0.75, p \leq 0.01$.

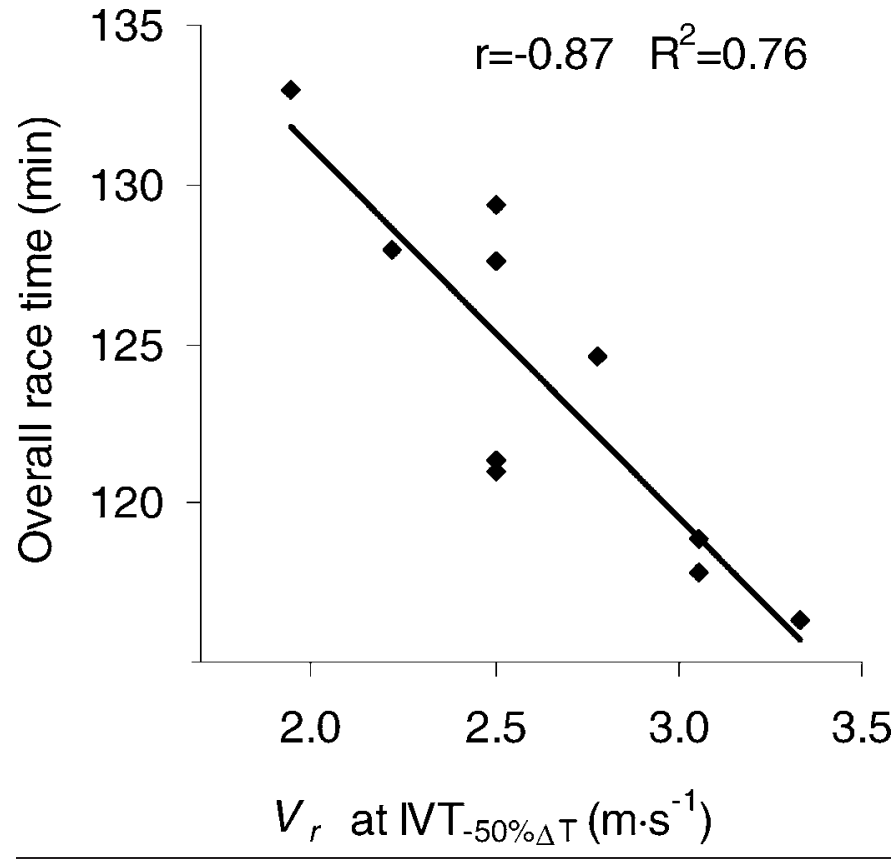

Figure 2. Relationship between overall race time in shortdistance triathlon (minutes) and running velocity at IVT $_{-50 \% \Delta \mathrm{T}}$.

$\left.72 \mathrm{~mL} \cdot \mathrm{kg}^{-1} \cdot \mathrm{min}^{-1}\right)$ reported by different investigators $(26$, 31) indicates that factors other than $\mathrm{V}_{2}$ max contribute more significantly to triathlon performance. In fact, the ventilatory threshold with the economy of motion (26) and, more recently, the peak treadmill running velocity associated with blood lactate concentration during steady-state cycling at $4 \mathrm{~W} \cdot \mathrm{kg}^{-1}$ body mass (28) have been proposed as predictive parameters of triathlon performance.

In the current study, IVT, also known as the first ventilatory threshold (9) and point of optimum ventilatory efficiency (16), and IAT, also known as the maximal lactate steady state (MLSS), cannot be directly compared with other studies because of different methods of threshold determination. As previously proposed, we used a 3 -minute incremental test for both IVT $(1,16)$ and IAT (1). These 2 thresholds indicate 2 different exercise intensities. The IVT indicates the upper limit of an almost exclusive aerobic exercise $(16,18,23)$ with a low blood lactate concentration (about $2 \mathrm{mmol} \cdot \mathrm{L}^{-1}$ ). The IAT indicates the highest power output at MLSS (1, 23, 33, 34), i.e., when the production and the removal of lactate are in equilibrium (at about $4 \mathrm{mmol} \cdot \mathrm{L}^{-1}$ ). A previous study in elite triathletes $\left(\mathrm{V}_{2} \max 71.8 \pm 7.6 \mathrm{~mL} \cdot \mathrm{kg}^{-1} \cdot \mathrm{min}^{-1}\right)$ estimated the MLSS by assessing the respiratory compen- sation threshold during an every 1-minute incrementload protocol (17). However, it was recently observed that respiratory compensation threshold (1-minute incremental protocol) overestimates the MLSS by $10 \% \mathrm{~V}_{2} \max (9)$. Conversely, the protocol we used can underestimate the MLSS by 5-7\% (1). Therefore, the treadmill running velocities we observed for IVT and IAT are lower than those reported by Hue (17). However, we found $V_{r}$ at IAT somewhat higher than that reported $\left(3.7-3.9 \mathrm{~m} \cdot \mathrm{s}^{-1}\right)$ by other authors (21) who estimated the lactate threshold using 4 different methods in nonelite triathletes $\left(\mathrm{V}_{2}\right.$ peak $56.6 \pm$ $\left.1.3 \mathrm{~mL} \cdot \mathrm{kg}^{-1} \cdot \mathrm{min}^{-1}\right)$.

Whereas different studies have reported the physiological characteristics $(3,25,28)$ and physiological factors or predictors of success in the Olympic triathlon $(8,17$, 28 ), we have focused on the relationship between race performance and the individual efficient power output for lactate removal. The present data show that performance in running and cycle segments was highly $(p<0.01)$ related to overall race time, as previously reported $(8,28)$. A certain relationship $(p<0.05)$ was also observed between swim time and overall race time. Different results in the literature could be due to the difference between performing the swimming segment in a pool or in the sea.

The studied recovery power outputs IVT $_{+50 \% \Delta \mathrm{T}}$, IVT, and $\mathrm{IVT}_{-50 \% \Delta \mathrm{T}}$ ) produced different lactate removal curves. The significant lower values of the lactate removal curve at $\mathrm{IVT}_{-50 \% \Delta \mathrm{T}}$ indicated that this was the most efficient individual power output for lactate removal.

The $\mathrm{V}_{\mathrm{r}}$ at different submaximal power outputs (IAT, $\mathrm{IVT}_{+50 \% \Delta \mathrm{T}}$, IVT, and $\mathrm{IVT}_{-50 \% \Delta \mathrm{T}}$ ) was related differently to split and overall race time. In particular, the $\mathrm{V}_{\mathrm{r}}$ at $\mathrm{IVT}_{-50 \% \Delta \mathrm{T}}$ was highly related to cycle, run, and overall race time $(r=-0.82, r=-0.93$, and $r=-0.87$, respectively; $p<0.01$ ). Also, the $\mathrm{V}_{\mathrm{r}}$ at IVT was highly related to cycle, run, and overall race time $(r=-0.81, r=-0.91$, and $r=-0.85$, respectively; $p>0.01$ ). In short-distance triathlon, Schabort and colleagues (28) reported that percent $\mathrm{V}_{2}$ max sustained at $15 \mathrm{~km} \cdot \mathrm{h}^{-1}$ was significantly correlated with a $10-\mathrm{km}$ running time $(r=0.83, p<0.01)$ and with the total time $(r=0.81, p<0.01)$.

The $\mathrm{VO}_{2}$ values at IAT, IVT related $(p<0.05)$ to split and overall race times. In particular, the $\mathrm{VO}_{2}$ at IAT was related to cycle, run, and overall race time ( $r=0.67, r=0.63, r=0.69$, respectively), and the $\mathrm{VO}_{2}$ at IVT was related to overall race time $(r=$ 0.64). The relationship between the $\mathrm{VO}_{2}$ at lactate threshold and the $40-\mathrm{km}$ cycle performance has been previously reported (7). Also, for overall successful performance, a high $\mathrm{VO}_{2}$ at ventilatory (8) and anaerobic (30) thresholds was reported.

The main finding of the present study was that $\mathrm{V}_{\mathrm{r}}$ at 
the power output of optimal lactate removal was the variable most related to overall race performance. In fact, the correlation coefficient of -0.87 and the $R^{2}$ of 0.76 determined by multiple stepwise linear regression indicated that $\mathrm{V}_{\mathrm{r}}$ at IVT $\mathrm{IV0 \% \Delta \textrm {T }}_{-5}$ alone could account for $76 \%$ of the variance in triathlon race time. Apparently such a relationship between individual overall performance and the running velocity at the optimal lactate removal power output has not yet been reported.

Previous studies have shown that blood lactate, although elevated after the swimming segment, decreases progressively during triathlon races (12), indicating the use of lactate as an energy substrate by exercising muscle. Lactate oxidation from active muscles has been described as the major pathway of lactate metabolism (6). Slow-twitch fibers of skeletal muscle are metabolically oxidative fibers that tend to consume lactate $(6,32)$, whereas fast-twitch fibers are glycolic anaerobic fibers that produce lactate (10). Which type of fiber is predominantly active during an exercise depends not only on the exercise intensity but also on the training status of the subject (13). A triathlete may have a high percentage of slowtwitch fibers as a consequence of metabolic adaptations induced by endurance training $(5,13)$.

\section{Practical Applications}

The present findings suggest that better overall race time is associated with a higher running velocity at which the subject has his most efficient lactate removal (IVT ${ }_{-50 \% \Delta \mathrm{T}}$ ). Having a high $\mathrm{V}_{\mathrm{r}}$ at IVT ${ }_{-50 \% \Delta \mathrm{T}}$ indicates that the triathlete has a high $\mathrm{V}_{\mathrm{r}}$ at IVT and little difference between IAT and IVT $(\Delta \mathrm{T})$. The shift towards a higher $\mathrm{V}_{\mathrm{r}}$ of IAT with a greater shift towards a high $V_{r}$ at IVT led to higher $V_{r}$ at IVT $_{-50 \% \Delta \mathrm{T}}$, the optimal $\mathrm{V}_{\mathrm{r}}$ for lactate removal, which was associated with better overall race performance.

These findings are of particular interest to coaches and triathletes and are especially relevant to designing proper training sessions. Previous studies have recommended high-intensity interval training as a viable method for improving endurance performance, suggesting also that regular assessments of training status and subsequent adjustments to the training program are required to maximize performance improvements $(20,29)$. Interval training alternates brief high-intensity exercise bouts with rest or relief periods. We suggest performing the relief periods at the exercise intensity corresponding to the individual optimal lactate removal, such as $\mathrm{Vr}$ at $\mathrm{IVT}_{-50 \% \Delta \mathrm{T}}$, in order to allow the next exercise bouts to continue with minimal fatigue and thus to increase the time to exhaustion. In addition, because a high $\mathrm{V}_{\mathrm{r}}$ at IVT $\mathrm{IV}_{-50 \% \Delta \mathrm{T}}$ is related to better overall triathlon race performance, we suggest that regular measurement of $V_{r}$ at IVT and IAT (from which $\mathrm{Vr}$ at $\mathrm{IVT}_{-50 \% \Delta \mathrm{T}}$ can be calculated) may be a useful method for monitoring training-induced adaptations and performance improvements in athletes who participate in Olympic triathlons.

During a triathlon, high levels of blood lactate were observed after the swimming and cycling segments (8.1 and $5.1 \mathrm{mmol} \cdot \mathrm{l}^{-1}$, respectively) (12). This may result in a significant power reduction on the subsequent cycling and running segments. In draft-legal triathlon, participants could adopt an individualized strategy because drafting results in a considerable lowering of power output (3). Moreover, race organizers may include 1 or several hill sections within each loop of the course, such that the race would involve a "stochastic" burst of very high power output interspersed with more submaximal exer- cise (25). The submaximal exercise would be performed at IVT $_{-50 \% \Delta \mathrm{T}}$ intensity, and the lactate removal would be optimized. In this sense, it is advantageous for athletes to have a high $\mathrm{V}_{\mathrm{r}}$ at IVT $\mathrm{IV}_{-50 \% \mathrm{~T}}$, the power output at which working muscles can efficiently oxidize lactate. In conclusion, individual overall race performance in short-distance triathlons is highly related to the athlete's $V_{r}$ at which the most efficient lactate removal $\left(\mathrm{IVT}_{-50 \% \Delta \mathrm{T}}\right.$ ) occurs.

\section{REFERENCES}

1. BALDARI, C., AND L. GUIDETTI. A simple method for individual anaerobic threshold as predictor of max lactate steady state. Med. Sci. Sports Exerc. 32:1798-1802. 2000.

2. Baldari, C., M. Videira, F. Madeira, J. Sergio, and L. Guidetti. Lactate removal during active recovery related to the individual anaerobic and ventilatory thresholds in soccer players. Eur. J. Appl. Physiol. 93: 224-230. 2004.

3. Bentley, D.J., G.P. Millet, V.E. Vleck, and L.R. McNaughton. Specific aspects of contemporary triathlon: Implication for physiological analysis and performance. Sports Med. 32:345-359. 2002.

4. Bishop, D. Evaluation of the Accusport lactate analyzer. Int. J. Sports Med. 22:525-530. 2001

5. Bonen, A. Lactate transporters (MCT proteins) in heart and skeletal muscles. Med. Sci. Sports Exerc. 32:778-789. 2000.

6. BRooks, G.A. Intra- and extra-cellular lactate shuttles. Med. Sci. Sport Exerc. 32:790-799. 2000.

7. Coyle, E.F., M.E. Feltner, S.A. Kautz, M.T. Hamilton, S.J. Montain, A.M. Baylor, L.D. Abraham, AND G.W. Petrek. Physiological and biomechanical factors associated with elite endurance cycling performance. Med. Sci. Sports Exerc. 23:93-107. 1991.

8. De Vito, G., M. Bernardi, E. Sproviero, and F. Figura. Decrease of endurance performance during Olympic triathlon. Int. J. Sports Med. 1: 24-28. 1995.

9. Dekerle J., B. Baron, L. Dupont, J. Vanvelcenaher, and P. Pelayo. Maximal lactate steady state, respiratory compensation threshold and critical power. Eur. J. Appl. Physiol. 89:281-288. 2003.

10. Donovan, C.M., AND M.J. PAGLIAssotTi. Quantitative assessment of pathways for lactate disposal in skeletal muscle fiber types. Med. Sci. Sports Exerc. 32:772-779. 2000.

11. Duncan, G.E., E.T. Howley, And B.N. Johnson. Applicability of $\mathrm{V}_{2} \max$ criteria: Discontinuous versus continuous protocols. Med. Sci. Sports Exerc. 29:273-278. 1997.

12. Farber, H.W., E.J. Schaefer, R. Franey, R. Grimaldi, And N.S. Hill. The endurance triathlon: Metabolic changes after each event and during recovery. Med. Sci. Sports Exerc. 23:959-965. 1991.

13. Gladden, L.B. Muscle as a consumer of lactate. Med. Sci. Sports Exerc. 32:764-771. 2000.

14. Gollnick, P.D., W.M. Boyly, AND D.R. Hodgson. Exercise intensity, training, diet and lactate concentration in muscle and blood. Med. Sci. Sports Exerc. 18:334-340. 1986.

15. Hogan, M.C., L.B. Gladden, S.S. Kurdak, and D.C. Poole. Increased [lactate] in working dog muscle reduces tension development independent of pH. Med. Sci. Sports Exerc. 27:371-377. 1995

16. HollmanN, W. 42 years ago: Development of the concepts of ventilatory and lactate threshold. Sports Med. 31:315-320. 2001.

17. HuE, O. Prediction of drafted-triathlon race time from submaximal laboratory testing in elite triathletes. Can. J. Appl. Physiol. 28:547-560. 2003.

18. Kindermann, W., G. Simon, AND J. KeUL. The significance of the aerobic-anaerobic transition for the determination of work load intensities during endurance training. Eur. J. Appl. Physiol. 42:25-34. 1979.

19. KLAUSEN, K., H.G. KNUTTGEN, AND H.V. Forster. Effect of pre-existing high blood lactate concentration on maximal exercise performance. Scand. J. Clin. Lab. Invest. 30:415-419. 1972.

20. LAURsen, P.B., AND D.G. Jenkins. The scientific basis for high-intensity interval training: Optimising training programmes and maximising performance in highly trained endurance athletes. Sports Med. 32:53-73. 2002.

21. MCGeheE, J.C., C.J. TANner, AND J.A. HoumarD. A comparison of methods for estimating the lactate threshold. J. Strength Cond. Res. 19:553558. 2005.

22. MCLELlan, T.M., AND J. S. SkinNER. Blood lactate removal during active recovery related to the aerobic threshold. Int. J. Sports Med. 3:224-229. 1982.

23. Meyer, T., A. Lucia, C.P. EARnest, And W. Kindermann. A conceptual framework for performance diagnosis and training prescription from submaximal gas exchange parameters: Theory and application. Int. J. Sports Med. 26:S38-S48. 2005. 
24. Meyer, T., R.C. Davison, And W. Kindermann. Ambulatory gas exchange measurements: Current status and future options. Int. J. Sports Med. 26:S19-S27. 2005.

25. Millet, G.P., P. Dreano, And D.J. Bently. Physiological characteristics of elite short- and long-distance triathletes. Eur. J. Appl. Physiol. 88:427430. 2003.

26. O'Toole, M.L., And P.S. Douglas. Applied physiology of triathlon. Sports Med. 19:251-267. 1995.

27. SAHLIN, K., AND J.M. REN. Relationship of contraction capacity to metabolic changes during recovery from a fatiguing contraction. J. Appl. Physiol. 67:648-654. 1989.

28. Schabort, E.J., S.C. Killian, A. St Clair Gibson, J.A. Hawley, AND T.D. NoAKES. Prediction of triathlon race time from laboratory testing in national triathletes. Med. Sci. Sports Exerc. 32:844-849. 2000.

29. Slattery, K.M., L.K. Wallace, A.J. MurPhy, and A.J. Coutts. Physiological determinants of three-kilometers running performance in experienced triathletes. J. Strength Cond. Res. 20:47-52. 2006.

30. Sleivert, G.G., AND D. S. Rowlands. Physical and physiological factors associated with success in triathlon. Sport Med. 22:8-18. 1996.
31. Sleivert, G.G., And H.A. Wenger. Physiological predictors of shortcurse triathlon performance. Med. Sci. Sports Exerc. 25:871-876. 1993.

32. Spriet, L.L., R.A. Howlett, AND G.J.F. Heigenhauser. An enzymatic approach to lactate production in human skeletal muscle during exercise. Med. Sci. Sports Exerc. 32:756-763. 2000.

33. Stegmann, H., W. Kindermann, And A. Schnabel. Lactate kinetics and individual anaerobic threshold. Int. J. Sports Med. 2:160-165. 1981.

34. Urhausen, A., B. Coen, B. Weiler, and W. Kindermann. Individual anaerobic threshold and maximum lactate steady state. Int. J. Sports Med. 14:134-139. 1993.

\section{Acknowledgments}

This research had financial support by the Insitituto Universitario di Scienze Motorie (IUSM).

Address correspondence to Dr. Carlo Baldari, carlo. baldari@iusm.it. 